\title{
Left and right compatibility of strict orders with fuzzy tolerance and fuzzy equivalence relations
}

\author{
B. De Baets ${ }^{1}$ L. Zedam, ${ }^{2}$ A. Kheniche ${ }^{3}$ \\ ${ }^{1}$ KERMIT, Department of Mathematical Modelling, Statistics and Bioinformatics, Ghent University, Belgium \\ ${ }^{2}$ LMPA, Department of Mathematics, Med Boudiaf University - Msila, Algeria \\ ${ }^{3}$ Department of Mathematics, Med Boudiaf University - Msila, Algeria
}

\begin{abstract}
The notion of extensionality of a fuzzy relation w.r.t. a fuzzy equivalence was first introduced by Höhle and Blanchard. Bělohlávek introduced a similar definition of compatibility of a fuzzy relation w.r.t. a fuzzy equality. In [14] we generalized this notion to left compatibility, right compatibility and compatibility of arbitrary fuzzy relations and we characterized them in terms of left and right traces introduced by Fodor. In this note, we will again investigate these notions, but this time we focus on the compatibility of strict orders with fuzzy tolerance and fuzzy equivalence relations.
\end{abstract}

Keywords: Compatibility, crisp strict order, fuzzy tolerance relation, fuzzy equivalence relation

\section{Introduction}

Equivalence relations and orderings are fundamental concepts of mathematics. Various generalizations of these notions to fuzzy set theory have already been proposed and successfully used. The notion of a fuzzy relation is one of the most important concepts in fuzzy set theory, and was introduced by Zadeh in the early years [16]. Fuzzy equalities, and the more general fuzzy equivalence relations, have become very popular in several fields of applications and play a fundamental role, as they allow to shape the universe of discourse or set of alternatives, by stating in a gradual and transitive way how alike elements or alternatives are.

The notion of right (resp. left) extensionality of a fuzzy order with an $L$-equality has been introduced by Höhle and Blanchard in [12]. This notion is equivalent to the notion of the compatibility as coined by Bělohlávek [3], and it is very useful for many applications in the lattice-theoretic approach to concept lattices $[1,2]$. In general, this notion of compatibility refers to the relationship between objects and their properties expressed by considered relation. Roughly speaking, compatibility expresses here that elements that are similar to related elements are related as well. For more details and properties about compatibility of fuzzy relations we refer to [14].

Another notion of compatibility has been introduced by Bodenhofer and colleagues $[5,6,7,8]$. Bodenhofer has defined the compatibility of a fuzzy equivalence relation with a crisp order as follows: the two outer elements of an ascending threeelement chain are at most as similar as any two elements of this chain. These two notions of compatibility are not equivalent.

In this work, we focus on the right (resp. left) compatibility introduced by Höhle and Blanchard in [12] and that is given by Bělohlávek [3]; we provide characterizations of the compatibility of a crisp strict order with fuzzy tolerance and fuzzy equivalence relations.

This work is organized as follows: Section 2 contains basic definitions of (complete) residuated lattices and fuzzy relations. In Section 3, we give useful notions about compatibility of fuzzy relations. In Section 4, we investigate the right (resp. left) compatibility of a strict order with fuzzy tolerance and fuzzy equivalence relations.

\section{Basic definitions}

In this section, we recall the basic definitions and properties of ordered sets, residuated lattices and fuzzy relations that will be needed throughout this work.

A partial order (order, for short) is a binary relation $\leqslant$ on a set $X$, which is reflexive $(a \leqslant a$, for any $a \in X)$, antisymmetric $(a \leqslant b$ and $b \leqslant a$ implies $a=b$, for any $a, b \in X)$ and transitive $(a \leqslant b$ and $b \leqslant c$ implies $a \leqslant c$, for any $a, b, c \in X)$. A set equipped with an order relation is called a partial ordered set (poset, for short). A strict order is a binary relation $<$ on a set $X$ that is irreflexive $(a<a$ does not hold for any $a \in X$ ) and transitive. The irreflexivity and the transitivity imply that a strict order is asymmetric, i.e. if $a<b$, then $b<a$ does not hold for any $a, b \in X$. A binary relation $\sim$ on a set $X$ is called an equivalence relation if it is reflexive, symmetric (i.e. $a \sim b$ implies that $b \sim a$ for any $a, b \in X)$ and transitive. 
If $\leqslant$ is an order, then the corresponding strict order $<$ is the irreflexive kernel given by

$$
a<b \text { if } a \leqslant b \text { and } a \neq b .
$$

Conversely, if $<$ is a strict order, then the corresponding order $\leqslant$ is the reflexive closure given by

$$
a \leqslant b \text { if } a<b \text { or } a=b .
$$

Two elements $x$ and $y$ of $X$ are called comparable if $x \leqslant y$ or $y \leqslant x$, otherwise they are called incomparable, and we write $x \| y$.

Consider a poset $(X, \leqslant)$. Then $x$ is called a lower cover of $y \in X$ (and $y$ is called an upper cover of $x)$ if $x<y$ and there exists no $z \in X$ such that $x<z<y$. In this case we write $x \ll y$. Elements $x$ and $y$ that satisfy $x \ll y$ or $y \ll x$ also called adjacent or neighbors. We will also use the notation $y \gg x$ to indicate that $x \ll y$.

A poset can be conveniently represented by a Hasse diagram, displaying the covering relation $\ll$. Note that $x<y$ if there is a sequence of connected lines upwards from $x$ to $y$. For more details about order, strict order and equivalence relations we refer to $[10,15]$.

A poset $(X, \leqslant)$ is called a lattice if any pair $x, y$ of elements of $X$ their supermum $x \vee y$ and infimum $x \wedge y$ exist. $(X, \leqslant)$ is called a complete lattice if any subset of $X$ has a supremum and an infimum.

A complete residuated lattice is an algebra $(L, \wedge, \vee, *, \rightarrow, 0,1)$ where $(L, \wedge, \vee, 0,1)$ is a complete lattice with the bottom element 0 and the top element $1,(L, *, 1)$ is a commutative monoid, $*$ and $\rightarrow$ called multiplication and residuum, satisfies the adjointness property: $a * b \leq c$ if and only if $a \leq b \rightarrow c$, for any $a, b, c \in L$. For more details about residuated and complete residuated lattices see, e.g., Bělohlávek [1, 2], Blyth and Janowitz [4], Ćirić [9], Hájek [11] and Schröder [15].

Throughout this work, unless otherwise stated, $L$ always denotes a (complete) residuated lattice.

Let $X$ be a nonempty universe and $L^{X}$ denote the set of all mappings from $X$ to $L$. A binary $L$ relation ( $L$-relation, for short) on $X$ is a mapping $R \in L^{X \times X}$. For any $x, y \in X$, the value $R(x, y)$ is called the degree of membership of $(x, y)$ in $R$. The transpose $R^{t}$ of $R$ is the $L$-relation on $X$ defined by $R^{t}(y, x)=R(x, y)$.

Let $R$ be an $L$-relation on a universe $X$. The following properties are of interest in this work (see, e.g., $[3,13,16])$ :

(i) Reflexivity: $R(x, x)=1$, for any $x \in X$,

(ii) Symmetry: $R(x, y)=R(y, x)$, for any $x, y \in$ $X$,

(iii) *-Transitivity: $R(x, y) * R(y, z) \leq R(x, z)$, for any $x, y, z \in X$,

(iv) Separability: $R(x, y)=1$ implies $x=y$, for any $x, y \in X$.
A reflexive and symmetric $L$-relation is called an $L$-tolerance relation. A $*$-transitive $L$-tolerance is called an $L$-equivalence relation. A separable $L$-equivalence on $X$ is called an $L$-equality on $X$. Note that the only $\{0,1\}$-equality on $X$ is precisely the usual equality (identity) $\operatorname{Id}_{X}$, i.e. $\operatorname{Id}_{X}(x, y)=1$ if $x=y$ and $\operatorname{Id}_{X}(x, y)=0$ if $x \neq y$. Therefore, the notion of $L$-equality is a natural generalization of the classical (bivalent) notion.

\section{Useful notions of compatibility of fuzzy relations}

In this subsection, useful notions and preliminary results about compatibility of fuzzy relations that will be used in the next sections are given.

Definition 1. [3] Let $X$ be a universe equipped with an $L$-equality $E$. An $L$-relation $R$ on a universe $X$ is compatible w.r.t. $E$ if

$$
R\left(x_{1}, y_{1}\right) * E\left(x_{1}, x_{2}\right) * E\left(y_{1}, y_{2}\right) \leq R\left(x_{2}, y_{2}\right),
$$

for any $x_{1}, x_{2}, y_{1}, y_{2} \in X$.

In [14], we generalized this definition of compatibility to arbitrary fuzzy relations.

Definition 2. [14] Let $R_{1}$ and $R_{2}$ be two $L$ relations on a universe $X$.

(i) $R_{1}$ is called left compatible with $R_{2}$, denoted $R_{1} \nabla_{l} R_{2}$, if the following inequality holds

$$
R_{1}(x, y) * R_{2}(x, z) \leq R_{1}(z, y),
$$

for any $x, y, z \in X$;

(ii) $R_{1}$ is called right compatible with $R_{2}$, denoted $R_{1} \nabla_{r} R_{2}$, if the following inequality holds

$$
R_{1}(x, y) * R_{2}(y, t) \leq R_{1}(x, t),
$$

for any $x, y, t \in X$;

(iii) $R_{1}$ is called compatible with $R_{2}$, denoted $R_{1} \nabla R_{2}$, if the following inequality holds

$$
R_{1}(x, y) * R_{2}(x, z) * R_{2}(y, t) \leq R_{1}(z, t),
$$

for any $x, y, z, t \in X$.

Lemma 1. [14] For any two $L$-relations $R_{1}$ and $R_{2}$ on a universe $X$, the following equivalences hold:

(i) $R_{1} \nabla_{l} R_{2}$ if and only if $R_{1}^{t} \nabla_{r} R_{2}$,

(ii) $R_{1} \nabla_{r} R_{2}$ if and only if $R_{1}^{t} \nabla_{l} R_{2}$,

(iii) $R_{1} \nabla R_{2}$ if and only if $R_{1}^{t} \nabla R_{2}$.

Proposition 1. [14] Let $R_{1}$ and $R_{2}$ be two $L$ relations on a universe $X$. Then, it holds that

(i) If $R_{1} \nabla_{l} R_{2}$ and $R_{1} \nabla_{r} R_{2}$, then $R_{1} \nabla R_{2}$;

(ii) If $R_{1} \nabla R_{2}$ and $R_{2}$ is reflexive, then $R_{1} \nabla_{l} R_{2}$ and $R_{1} \nabla_{r} R_{2}$. 
For more properties of the crisp relations $\nabla_{r}, \nabla_{l}$ and $\nabla$, we refer to [14].

Next, we introduce some crisp relations, which shall be used to characterize the left and right compatibility of strict orders with $L$-tolerance and $L$ equivalence relations. Let $(X,<)$ be a strictly ordered set, for any $x, y \in X$,

(i) $x \approx_{<}^{l} y$ if and only if $z<x \Leftrightarrow z<y$ for any $z \in X \backslash\{x, y\}$

(ii) $x \approx_{<}^{r} y$ if and only if $x<z \Leftrightarrow y<z$ for any $z \in X \backslash\{x, y\}$

Remark 1. Note that $\approx_{<}^{l}=\approx_{<^{t}}^{r}$ and $\approx_{<}^{r}=\approx_{<^{t}}^{l}$.

The following proposition is immediate.

Proposition 2. Let $(X,<)$ be a strictly ordered set. Then $\approx_{<}^{l}$ and $\approx_{<}^{r}$ are reflexive and symmetric relations.

Example 1. Let $(X, \leqslant)$ be a poset given by the following Hasse diagram.

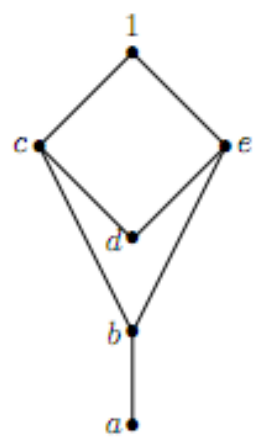

Figure 1: A poset $(X, \leqslant)$ with $X=\{a, b, c, d, e, 1\}$.

In this example,

(i) $a \approx_{<}^{l} b, a \approx_{<}^{l} d$ and $c \approx_{<}^{l} e$;

(ii) $a \approx_{<}^{r} b, b \approx_{<}^{r} d, c \approx_{<}^{r} e, c \approx_{<}^{r} 1$ and $e \approx_{<}^{r} 1$.

In addition to the elements coming from the reflectivity and the symmetry of $\approx_{<}^{l}$ and $\approx_{<}^{r}$ there are no other cases.

Useful properties of the relations $\approx_{<}^{r}, \approx_{<}^{l}$ and $\approx_{<}$ are given in the following propositions.

Proposition 3. Let $(X,<)$ be a strictly ordered set and $x, y \in X$ such that $x \neq y$. Then, it holds that

(a) If $x \approx_{<}^{r} y$, then $x \ll y, y \ll x$ or $x \| y$,

(b) If $x \approx_{<}^{l} y$, then $x \ll y, y \ll x$ or $x \| y$.

Proposition 4. Let $(X,<)$ be a strictly ordered set and $x, y \in X$. Then, it holds that (a) Exactly one of the following statements holds:
(i) $x=y$,
(ii) $x \approx_{<}^{r} y$ and $x \ll y$,
(iii) $x \approx_{<}^{r} y$ and $y \ll x$,
(iv) $x \approx_{<}^{r} y$ and $x \| y$,
(v) $x \not_{<}^{r} y$.

(b) Exactly one of the following statements holds:
(i) $x=y$,
(ii) $x \approx_{<}^{l} y$ and $x \ll y$,
(iii) $x \approx_{<}^{l} y$ and $y \ll x$,
(iv) $x \approx_{<}^{l} y$ and $x \| y$,
(v) $x \not_{<}^{l} y$.

For the tolerance relations $\approx_{<}^{r}$ and $\approx_{<}^{l}$ of a strictly ordered set $(X,<)$, we introduce the following relations $\approx_{\|}^{r}$ and $\approx_{\|}^{l}$ on $X: x \approx_{\|}^{r} y$ if and only if $x \approx_{<}^{r} y$ and $x \| y$, for any $x, y \in X$,

$$
x \approx_{\|}^{r} y \text { if and only if } x \approx_{<}^{r} y \text { and } x \| y
$$

and

$$
x \approx_{\|}^{l} y \text { if and only if } x \approx_{<}^{l} y \text { and } x \| y,
$$

for any $x, y \in X$.

Proposition 5. The relations $\approx_{\|}^{r}$ and $\approx_{\|}^{l}$ are transitive.

Remark 2. In general, the relations $\approx_{<}^{r}$ and $\approx_{<}^{l}$ are not transitive (see Example 1 ).

From Propositions 2 and 5, we derive the following corollary.

Corollary 1. Let $(X,<)$ be a strictly ordered set. Then, it holds that

(i) $\approx_{<}^{r}$ and $\approx_{<}^{l}$ are tolerance relations on $X$,

(ii) $\approx_{<}^{r}$ and $\approx_{<}^{l}$ are equivalence relations on the set of incomparable elements of $(X,<)$.

\section{Left and right compatibility of strict orders with fuzzy tolerance and fuzzy equivalence relations}

In this section, we shall characterize the $L$-tolerance and $L$-equivalence such that a crisp strict order is compatible with them. First, we need the following notation.

Notation 1. The symbol $\tau$ stands for truth value, i.e., for a proposition $p, \tau(p)=1$ means that $p$ is true and $\tau(p)=0$ means that $p$ is false.

Let $<$ be a crisp strict order on a universe $X$ and $E$ be an $L$-relation on $X$, consider the right compatibility, the left compatibility and the compatibility of the crisp strict order $<$ with the $L$-relation $E$ as follows: 
(i) $<$ is called right compatible with $E$, if it holds that

$$
\tau(x<y) * E(y, z) \leq \tau(x<z)
$$

for any $x, y, z \in X$

(ii) $<$ is called left compatible with $E$, if it holds that

$$
\tau(x<y) * E(x, z) \leq \tau(z<y),
$$

for any $x, y, z \in X$

(iii) $<$ is called compatible with $E$, if it holds that

$$
\tau(x<y) * E(x, z) * E(y, t) \leq \tau(z<t),
$$

for any $x, y, z \in X$.

The following lemma is immediate.

Lemma 2. Let $(X,<)$ be a strictly ordered set. Then it holds that

(i) $<$ is right compatible with $\approx_{<}^{l}$;

(ii) $<$ is left compatible with $\approx_{<}^{r}$.

In the following theorem, we will show that the right (resp. left) compatibility can be expressed in terms of $\approx_{<}^{l}$ and $\approx_{<}^{r}$ introduced in the above section.

Theorem 1. Let $(X,<)$ be a strictly ordered set and $E$ be an $L$-tolerance relation on $X$. Then the following equivalences hold

(i) $<$ is right compatible with $E$ if and only if $E \subseteq \approx_{<}^{l}$

(ii) $<$ is left compatible with $E$ if and only if $E \subseteq \approx_{<}^{r}$.

\section{Remark 3.}

(i) $\approx_{<}^{l}$ is the greatest $L$-tolerance relation on $X$ such that $<$ is right compatible with it,

(ii) $\approx_{<}^{r}$ is the greatest $L$-tolerance relation on $X$ such that $<$ is left compatible with it.

Theorem 2. Let $(X,<)$ be a strictly ordered set and $E$ be an $L$-tolerance relation on $X$. Then, it holds that

(i) < is right compatible with $E$ if and only if for any $x, y \in X$,

$$
E(x, y)= \begin{cases}1 & \text { if } x=y \\ \alpha_{r}(x, y) & \text { if }\left(x \approx_{<}^{l} y \text { and } x \ll y\right) \\ & \text { or }\left(x \approx_{<}^{l} y \text { and } y \ll x\right) \\ \beta_{r}(x, y) & \text { if } x \approx_{<}^{l} y \text { and } x \| y \\ 0 & \text { if } x \not_{<}^{l} y\end{cases}
$$

(1) $\alpha_{r}(x, y)=\alpha_{r}(y, x)$ if $x \approx_{<}^{l} y$ and $(x \ll y$ or $y \ll x)$,

(2) $\beta_{r}(x, y)=\beta_{r}(y, x)$ if $x \approx_{<}^{l} y$ and $x \| y$.

(ii) $<$ is left compatible with $E$ if and only if for any $x, y \in X$,

$$
E(x, y)= \begin{cases}1 & \text { if } x=y \\ \alpha_{l}(x, y) & \text { if }\left(x \approx_{<}^{r} y \text { and } x \ll y\right) \\ & \text { or }\left(x \approx_{<}^{r} y \text { and } y \ll x\right) \\ \beta_{l}(x, y) & \text { if } x \approx_{<}^{r} y \text { and } x \| y \\ 0 & \text { if } x \not_{<}^{r} y\end{cases}
$$

where $\alpha_{l}(x, y), \beta_{l}(x, y) \in L$ and satisfying:

(1) $\alpha_{l}(x, y)=\alpha_{l}(y, x)$ if $x \approx_{<}^{r} y$ and $(x \ll y$ or $y \ll x)$,

(2) $\beta_{l}(x, y)=\beta_{l}(y, x)$ if $x \approx_{<}^{r} y$ and $x \| y$.

\section{Remark 4.}

(i) If $\alpha_{r}(x, y)=\beta_{r}(x, y)=0$, for any $x, y \in X$, then $E$ is the crisp equality.

(ii) If $\alpha_{r}(x, y)=\beta_{r}(x, y)=1$, for any $x, y \in X$, then $E=\approx_{<}^{l}$.

In the following proposition, we will show that given additional conditions on $\alpha_{r}(x, y), \beta_{r}(x, y)$, $\alpha_{l}(x, y)$ and $\beta_{l}(x, y)$, the $L$-tolerance relations $(7)$ and (8) given in the above Theorem ?? are $L$ equivalence relations.

Proposition 6. Let $(X,<)$ be a strictly ordered set. Then, it holds that

(i) The $L$-relation $E$ defined by equation (7) where $\alpha_{r}(x, y), \beta_{r}(x, y) \in L$ satisfy the following conditions:

(1) $\alpha_{r}(x, y)=\alpha_{r}(y, x)$ if $x \approx_{<}^{l} y$ and $(x \ll y$ or $y \ll x)$,

(2) $\alpha_{r}(x, y) * \alpha_{r}(y, z) \leq \beta_{r}(x, z)$ if $\left(x \approx_{<}^{l} y\right.$ and $\left.y \ll x\right)$ and $\left(y \approx_{<}^{l} z\right.$ and $\left.y \ll z\right)$, and $\alpha_{r}(x, y) * \alpha_{r}(y, z)=0$, otherwise,

(3) $\alpha_{r}(x, y) * \beta_{r}(y, z) \leq \alpha_{r}(x, z)$ if $\left(x \approx_{<}^{l} y\right.$ and $\left.x \ll y\right)$, and $\alpha_{r}(x, y) * \beta_{r}(y, z)=0$ if $x \approx_{<}^{l} y$ and $y \ll x$,

(4) $\beta_{r}(x, y)=\beta_{r}(y, x)$ if $x \approx_{<}^{l} y$ and $x \| y$,

(5) $\beta_{r}(x, y) * \beta_{r}(y, z) \leq \beta_{r}(x, z)$.

is an $L$-equivalence relation on $X$.

where $\alpha_{r}(x, y), \beta_{r}(x, y) \in L$ and satisfying: 
(ii) The $L$-relation $E$ defined by by equation (8) where $\alpha_{l}(x, y), \beta_{l}(x, y) \in L$ satisfy the following conditions:

(1) $\alpha_{l}(x, y)=\alpha_{l}(y, x)$ if $x \approx_{<}^{r} y$ and $(x \ll y$ or $y \ll x)$,

(2) $\alpha_{l}(x, y) * \alpha_{l}(y, z) \leq \beta_{l}(x, z)$ if

$\left(x \approx_{<}^{r} y\right.$ and $\left.x \ll y\right)$ and

$\left(y \approx_{<}^{r} z\right.$ and $\left.z \ll y\right)$,

and $\alpha_{l}(x, y) * \alpha_{l}(y, z)=0$, otherwise,

(3) $\alpha_{l}(x, y) * \beta_{l}(y, z) \leq \alpha_{l}(x, z)$ if

$\left(x \approx_{<}^{r} y\right.$ and $\left.y \ll x\right)$, and

$\alpha_{l}(x, y) * \beta_{l}(y, z)=0$ if $x \approx_{<}^{r} y$ and $x \ll y$,

(4) $\beta_{l}(x, y)=\beta_{l}(y, x)$ if $x \approx_{<}^{r} y$ and $x \| y$,

(5) $\beta_{l}(x, y) * \beta_{l}(y, z) \leq \beta_{l}(x, z)$.

is an $L$-equivalence relation on $X$.

From Theorem 2 and Proposition 6, we obtain the following characterization theorem.

Theorem 3. Let $(X,<)$ be a strictly ordered set and $E$ be an $L$-equivalence relation on $X$. Then, it holds that

(i) $<$ is right compatible with $E$ if and only if $E$ takes the form (7).

(ii) < is left compatible with $E$ if and only if $E$ takes the form (8).

\section{References}

[1] R. Bělohlávek, Fuzzy Relational Systems: Foundations and Principles, Kluwer Academic Publishers/Plenum Publishers, New York, 2002.

[2] R. Bělohlávek, Some properties of residuated lattices, Czech. Math. J. 53 (2003) 161-171.

[3] R. Bělohlávek, Concept lattices and order in fuzzy logic, Ann. Pure Appl. Logic 128 (2004) $277-298$.

[4] T.S. Blyth, M.F. Janowitz, Residuated Theory, Pergamon Press, London, 1972.

[5] U. Bodenhofer, A new approach to fuzzy orderings, Tatra Mt. Math. Publ. 16 (1999) 1-9.

[6] U. Bodenhofer, A Similarity-Based Generalization of Fuzzy Orderings, Schriftenreihe der Johannes-Kepler-Universität Linz, Vol. C26, Universitätsverlag Rudolf Trauner, 1999.

[7] U. Bodenhofer, Representations and constructions of similarity-based fuzzy orderings, Fuzzy Sets and Systems 137 (2003) 113-136.

[8] U. Bodenhofer, B. De Baets, J. Fodor, A compendium of fuzzy weak orders: Representations and constructions, Fuzzy Sets and Systems 158 (2007) 811-829.

[9] M.D. Ćirić, J. Ignjatović, S. Bogdanović, Fuzzy equivalence relations and their equivalence classes, Fuzzy Sets and Systems 158 (2007) 12951313 .

[10] B.A. Davey, H.A. Priestley, Introduction to Lattices and Order, Second ed., Cambridge University Press, Cambridge, 2002.

[11] P. Hájek, Metamathematics of Fuzzy Logic, Trends in Logic, Vol. 4, Kluwer Academic Publishers, Dordrecht, 1998.

[12] U. Höhle and N. Blanchard. Partial ordering in L-underdeterminate sets. Inform. Sci. 35 (1985) 133-144.

[13] U. Höhle, Fuzzy equalities and indistinguishability, Proc. EUFIT'93, 1 (1993) 358-363.

[14] A. Kheniche, B. De Baets, L. Zedam, Compatibility of fuzzy relations, Submitted.

[15] B. S. Schröder, Ordered sets, Birkhauser Boston; First edition, December 6, 2002.

[16] L.A. Zadeh, Similarity relations and fuzzy orderings, Info. Sci. 3 (1971) 177-200. Info. Sci., 3(1971) 177-200. 